\title{
Start-to-end modelling of a mode-locked optical klystron free electron laser amplifier
}

\author{
D. J. Dunning, ${ }^{1,2, a)}$ B. W. J. Mc Neil, ${ }^{1, b)}$ N. R. Thompson, ${ }^{1,2, c)}$ and P. H. Williams ${ }^{2, d)}$ \\ ${ }^{1}$ University of Strathclyde (SUPA), Glasgow G4 ONG, United Kingdom \\ ${ }^{2} A S T e C$, Daresbury Laboratory, Warrington WA4 4AD, United Kingdom
}

(Received 26 April 2011; accepted 3 June 2011; published online 21 July 2011)

A free electron laser (FEL) in a mode-locked optical klystron (MLOK) configuration is modelled using start-to-end simulations that simulate realistic electron beam acceleration and transport before input into a full three-dimensional FEL simulation code. These simulations demonstrate that the MLOK scheme is compatible with the present generation of radiofrequency accelerator designs. A train of few-optical cycle pulses is predicted with peak powers similar to those of the equivalent conventional FEL amplifier. The role of electron beam energy modulation in these results is explained and the limitations of some simulation codes discussed. It is shown how seeding the FEL interaction using a High Harmonic seed laser can improve the coherence properties of the output. (C) 2011 American Institute of Physics. [doi:10.1063/1.3605027]

\section{INTRODUCTION}

The successful commissioning of the Linac Coherent Light Source (LCLS) x-ray free electron laser amplifier ${ }^{1}$ has introduced a new era of short wavelength free electron laser (FEL) facilities ${ }^{2}$ enabling unprecedented high brightness spatial resolution at the atomic scale $\sim 1 \AA$. In such FELs, a relativistic electron beam is injected into a magnetic undulator which causes the electrons to oscillate transversely to their direction of propagation along an undulator axis of period $\lambda_{u}$. The interaction of these undulating electrons with the radiation they emit results in a collective instability which bunches the electrons at a resonant wavelength $\lambda_{r}$, causing them to emit coherently at that wavelength. ${ }^{2}$ An initially small light signal of power $P_{0}$ is exponentially amplified along the $z$ axis of the undulator as $P(z) \approx P_{0} / 9 \exp \left(\sqrt{3} z / l_{g}\right)$ until saturation when the electrons begin to de-bunch. The nominal gain length of the collective instability $l_{g}=\lambda_{u} / 4 \pi \rho$, determines the rate of amplification of both the electron bunching and the radiation power and also the efficiency of the amplifier via $\rho$, the FEL parameter. ${ }^{3}$ Within the electron pulse, a radiation wavefront will propagate ahead of the copropagating electrons by one cooperation length $l_{c}=\lambda_{r} / 4 \pi \rho$ in one gain length through the undulator. ${ }^{4}$ Thus, the cooperation length also determines the coherence length $l_{c o h}$ of the radiation field with $l_{c o h} \approx l_{c}$. At short x-ray wavelengths, there are presently no external seed fields available for amplification and the FEL amplifier starts from noise. When the electron pulse lengths $l_{b}$ are long with respect to the cooperation length, $l_{b} \gg l_{c}$, many autonomous regions evolve from the noise that are uncorrelated in phase giving a "spikey" output with poor longitudinal (temporal) coherence. Each spike is of width $\sim l_{c}$

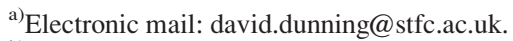

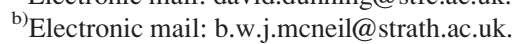

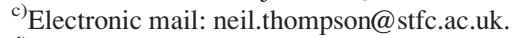

${ }^{\mathrm{d})}$ Electronic mail: peter.williams@stfc.ac.uk.
}

with spike separation of $\sim 2 \pi l_{c}{ }^{5}$ For shorter electron pulses $l_{b}$ $\lesssim 2 \pi l_{c}$, it is possible to have a single spike output of width $\sim l_{c}$. Thus, the cooperation length sets an approximate lower limit for the radiation pulse duration for unmodified FEL amplifier operation. Several schemes have been proposed to generate such short isolated radiation pulses from an FEL amplifier (see e.g., Refs. 6-8 and references therein), all with output pulse durations $\gtrsim l_{c} / c$, which in the x-ray gives typical minimum pulse durations $\approx 200$ as. Other methods are, therefore, required to achieve shorter durations towards the atomic unit of time of 24 as, which would allow interrogation and possible control of events at both the spatial and temporal resolution of the atom..$^{9,10}$

Two methods have been suggested that may achieve this goal. In Ref. 11, a modified echo enhanced harmonic generation scheme uses laser induced electron beam modulation and magnetic chicane dispersion to generate a single 20 as full width at half-maximum (FWHM) pulse of peak power $180 \mathrm{MW}$ at wavelength of $1 \mathrm{~nm}$. The second method is that of the mode locked optical klystron, ${ }^{12-14}$ which uses a method with similarities to mode generation and locking in conventional cavity oscillator lasers. Initial simulations predict that a MLOK FEL can generate a train of 23 as (FWHM) pulses, separated by 150 as, with peak powers of $\sim 5 \mathrm{GW}$ at a wavelength of $1.5 \AA$.

In a normal FEL amplifier, a resonant radiation wavefront propagates, or "slips," one resonant wavelength $\lambda_{r}$ over an electron in one undulator period. ${ }^{2}$ In the MLOK, additional slippage is introduced between the electrons and radiation by a periodic series of chicanes that delays the electrons. The FEL interaction then occurs in a periodic series of undulator-chicane modules. This creates radiation modes centred about the resonant wavelength and separated in frequency by $\Delta \omega=2 \pi c / s$ where $s$ is the total relative slippage between electrons and radiation in one undulator-chicane module. Despite operating as an amplifier, the MLOK method is similar to the process of mode-locking in conventional cavity 


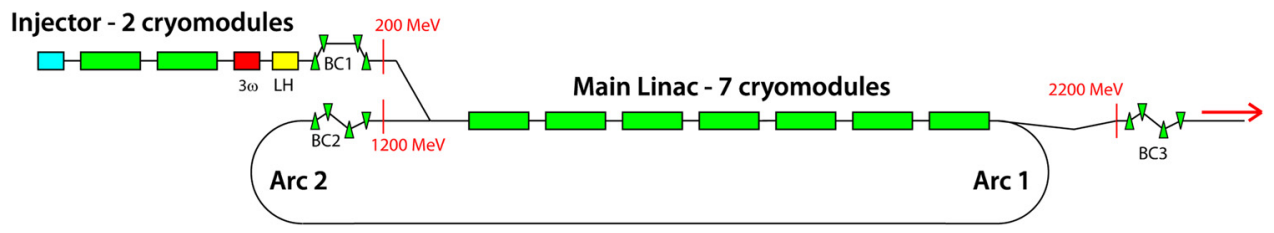

FIG. 1. (Color online) Schematic layout of the NLS recirculating accelerator. The $200 \mathrm{MeV}$ injector includes a third harmonic cavity (3 $\omega$ ), laser heater (LH), and bunch compression chicane (BC1). Two passes of a $1 \mathrm{GeV}$ main linac and two further bunch compression stages (BC2 and $\mathrm{BC} 3)$ follow.

oscillator lasers, where the slippage distance $s$ is equivalent to the round-trip cavity length of the conventional laser. Locking between modes is achieved, again in a manner similar to conventional lasers, by introducing a modulation at the mode spacing $\Delta \omega$. This, essentially optical method, results in the generation of a train of short pulses of duration $\ll l_{c} / c$, uniformly spaced at the modulation period.

In this paper, the MLOK method is modelled using a realistic electron beam generated by a full start-to-end simulation to demonstrate its compatibility with the predicted output from present generation accelerator designs for $\sim 1$ $\mathrm{nm}$ FEL output wavelength. The role of energy modulation of the electron beam to lock the modes is described in more detail than previously, in particular by considering the amplification of a high harmonic seed source. Amplification of a high harmonic $(\mathrm{HH})$ source has previously been investigated using simulations in the absence of any beam energy modulation $^{14}$ where a slow broadening of the individual radiation pulses was observed in simulations as the interaction progressed through the MLOK system. Some electron beam dispersive effects that are not included in the simulation model are identified, and the potential effects and benefits discussed.

\section{START-TO-END MODELLING}

The MLOK method is modelled in full three-dimensional FEL simulations using an electron distribution tracked through full start-to-end accelerator simulations of a superconducting re-circulating linac design. It is emphasised that the MLOK method is independent of the choice of accelerator configuration: the aim here is to demonstrate that the MLOK FEL is compatible with present accelerator designs. An overview of the method of generation of the electron distribution used in the simulations is given, and full threedimensional modelling of a MLOK FEL starting from noise is presented.

\section{A. Accelerator modelling}

An electron distribution for use in FEL modelling was generated in start-to-end simulations of an accelerator design $^{15}$ for the UK New Light Source (NLS) project. The design comprises of a $200 \mathrm{MeV}$ injector followed by two recirculation passes of a $1 \mathrm{GeV}$ main linac. The photoinjector $^{16}$ consists of a normal conducting L-band $(1.3 \mathrm{GHz})$ photocathode gun and a superconducting L-band $(1.3 \mathrm{GHz})$ cavity module and was simulated, for these lower energies, by using the simulation code ASTRA. ${ }^{17}$ The electron distribution generated by ASTRA was imported into the Elegant ${ }^{18}$ code for tracking through the accelerator lattice to the FEL entrance. The accelerator design is a two-pass recirculating machine based on nine TESLA-type accelerating cryomodules with longitudinal bunch compression carried out in three dedicated bunch compression stages. Following the linac are a beam switchyard, collimation, and diagnostic tomography sections. A laser heater is used to mitigate the effects of Coherent Synchrotron Radiation-induced microbunching. Figure 1 shows a schematic layout of the design.

The properties of the tracked electron distribution at the entrance to the FEL are given in Figure 2. The longitudinal phase space, current profile, slice emittance, and slice energy spread, binned in 1 fs slices, are shown. Peak current of $>1$ $\mathrm{kA}$ is achieved, but with some variation over 100 fs. Slice emittances of less than $0.4 \mathrm{~mm}$-mrad are attained. The slice energy spread is below $2 \times 10^{-4}$ for most of the bunch. A region of duration $\sim 100$ fs centred around the peak of the electron bunch current was selected from this distribution for FEL simulations. The rountine elegant2genesis ${ }^{19}$ was used to convert the electron distribution output from Elegant into an input distribution for the three dimensional FEL simulation code Genesis 1.3. ${ }^{20}$

\section{B. The mode locked optical klystron FEL amplifier}

The MLOK FEL amplifier ${ }^{12,14}$ uses similarities from mode-locking in conventional lasers to generate a train of short, uniformly spaced pulses, with individual pulse lengths significantly less than the cooperation length, $l_{c}$. An equally spaced modal structure is generated in the spectrum by periodically delaying the electron bunch using magnetic chicanes inserted between the undulator modules. The total relative slippage of the radiation through the electron bunch per undulator-chicane module is $s=l+\delta$, where $l=N_{u} \lambda_{r}$ is the slippage occurring in the undulator module of $N_{u}$ periods and $\delta$ that within the chicane. The slippage enhancement factor is defined as $S_{e}=s / l$. For $S_{e}>1$, the spectrum takes the form of a frequency comb modulated by the sinc-function envelope of the single undulator module spectrum centered at the resonant FEL frequency, $\omega_{r}$. The mode separation of the frequency comb is $\Delta \omega=2 \pi / T_{s}$, where $T_{s}=s / c$ is the time taken for radiation to propagate the slippage length. This mode spacing, therefore, determines the number of modes falling under the central envelope of the single undulator module spectrum, $N_{0}=2 S_{e}-1$.

In Ref. 12, the introduction of a sinusoidal modulation of the electron beam energy, of frequency equal to the mode spacing, causes the equally spaced modes to develop sidebands that overlap with neighbouring modes allowing modelocking to occur. This phase matches the modes to generate a temporal train of uniformly spaced short pulses. The effect 

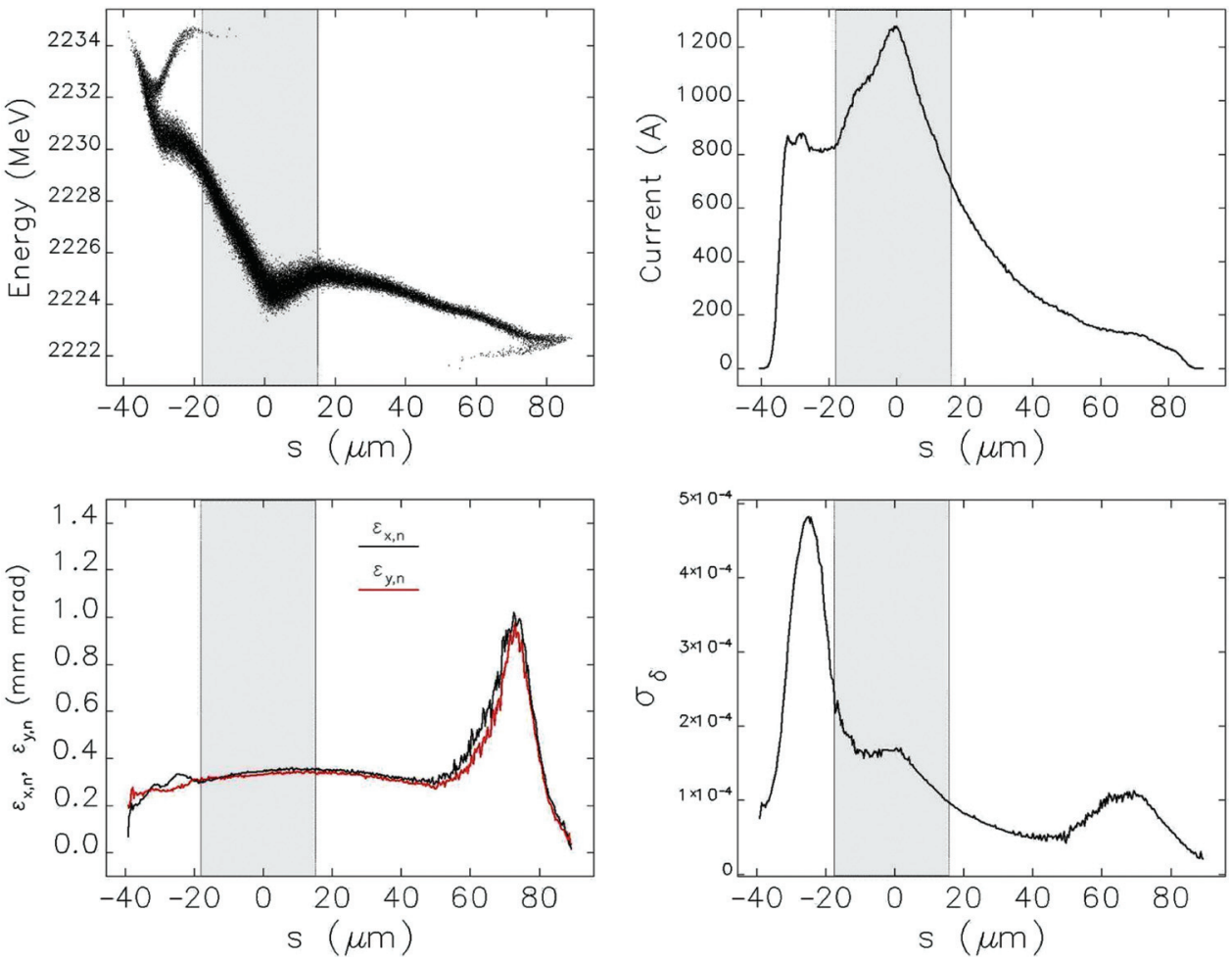

FIG. 2. (Color online) Bunch properties in $1 \mathrm{fs}$ bins on entrance to the modulator prior to entry to the MLOK FEL: Top left-longitudinal phase space, Top right-current profile, Bottom leftslice emittance, and Bottom right-slice energy spread. The region selected for FEL simulations is highlighted. of this energy modulation is considered in more detail in the following work.

The exemplar system used here has a resonant FEL wavelength of $\lambda_{r}=1.24 \mathrm{~nm}$, corresponding to the highest photon energy of the designs for the NLS FEL proposal. ${ }^{21}$ Undulator modules of $N_{u}=8$ periods were chosen so that $l=8 \lambda_{r}$, and the relative electron beam/radiation slippage induced by the dispersive effects of each chicane placed between the undulator modules was set to $\delta=23 \lambda_{r}$, giving a slippage enhancement factor of $S_{e}=3.9$.

\section{Modelling the MLOK FEL interaction}

The three-dimensional FEL code Genesis 1.3 (Ref. 20) was used to model the FEL interactions. A short modulator section was used to introduce a sinusoidal electron energy modulation in the tracked electron distribution of Fig. 2, of scaled amplitude $\gamma_{m}$ about the mean energy $\gamma_{0} \approx 4356$ and of period $s$ to match the total slippage per undulator-chicane module. The modulation amplitude was varied over a range of values and a value of $\gamma_{m} / \gamma_{0}=0.02 \%$ was chosen. The modulation amplitude that optimised the generation of the narrowest pulses was $\gamma_{m} / \gamma_{0} \approx 0.05 \%$, however, as discussed in Sec. IV, the effects of larger energy modulations may not be modelled sufficiently well by the current state of the art computer simulation codes like Genesis 1.3. Details of the effects of varying the modulation amplitude are presented and discussed in Sec. III.

The modular undulator-chicane structure was also modelled using Genesis 1.3, using the energy modulated tracked electron distribution. No seed was used so that the amplification process started from self-amplification of spontaneous emission $(\mathrm{SASE}){ }^{3}$ The radiation output power close to saturation after 120 undulator-chicane modules is shown in Fig. 3 and includes details of the initial electron beam energy and current profile. The total undulator length to saturation is $\sim 33 \mathrm{~m}$ and yields peak powers of $\sim 1 \mathrm{GW}$ similar to the results for a FEL amplifier operating in SASE mode using the same parameters. The peak of the radiation power occurs in the high current, low energy chirp section of the electron bunch. The pulse train retains the effects of SASE noise but
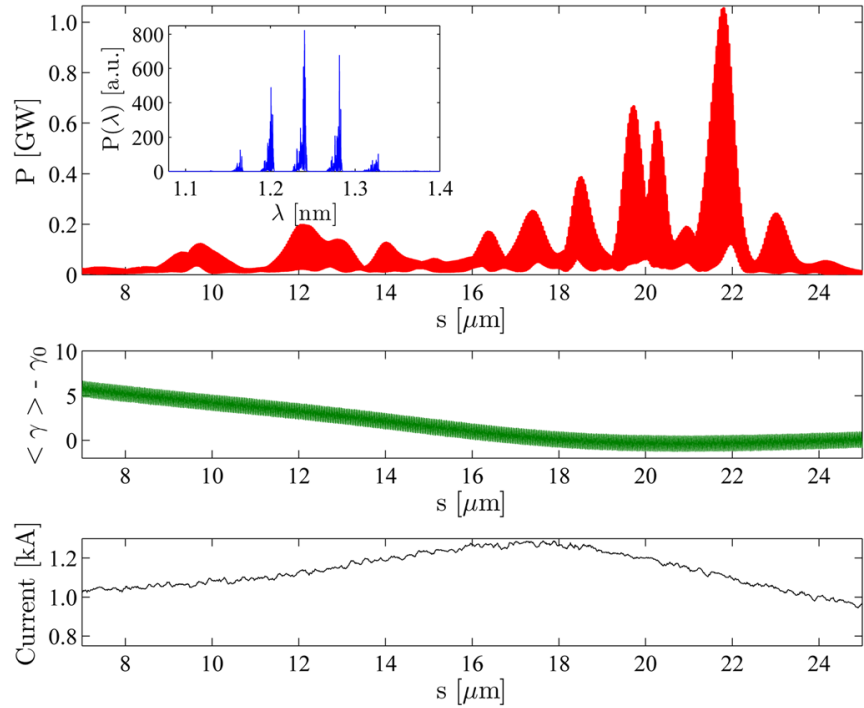

FIG. 3. (Color online) Top-radiation pulse output power close to saturation (120 modules) and the corresponding radiation spectrum (inset); Middle - the initial energy modulated electron beam energy; and Bottomelectron beam current profile. 

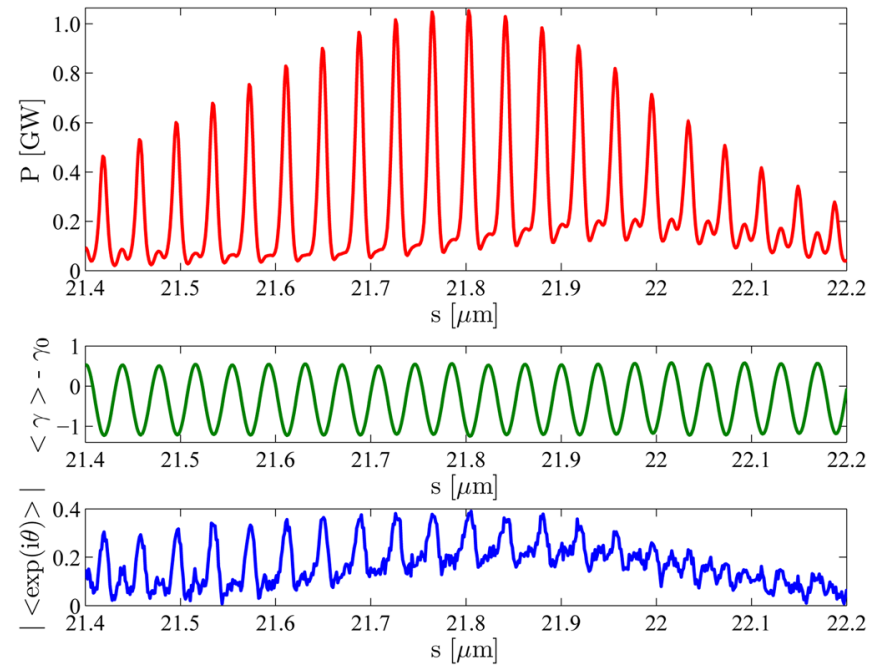

FIG. 4. (Color online) Top-radiation power at the centre of the 120th undulator module; Middle-slice-averaged electron beam energy offset from the resonant energy (in terms of electron rest mass energy); Bottomelectron beam bunching at the centre of the 120th undulator module. The radiation pulses align with the positions of minimum energy gradient.

with a longer envelope modulation. This longer modulation occurs as the cooperation length is increased by the slippage enhancement factor: $S_{e} \times l_{c}{ }^{12}$ Analysis of the radiation phase also shows that the coherence in the pulse train is extended by the same factor so that the coherence length $l_{c o h} \sim S_{e} \times l_{c}$. The energy chirp along the electron bunch results in a corresponding chirp in resonant radiation wavelength along the pulse train.

Fig. 4 shows a more detailed plot of the radiation power profile at the centre of the 120th undulator module, together with the electron beam energy and the modulus of the complex bunching parameter, $b=\left\langle e^{i \theta}\right\rangle$. The individual pulses within the train have durations $\sim 30$ as FWHM, with the number of optical cycles corresponding to approximately the number of undulator periods per module. The radiation pulse alignment relative to the modulated electron beam can also be observed. The radiation pulse trains align with either the minimum or maximum of the sinusoidal energy modulation. It is intuitively reasonable that these regions of minimum energy gradient appear to preferentially support the FEL interaction, allowing the radiation pulses to develop. Two pulses at the minima and maxima of the energy modulation, therefore, develop per modulation period, and two separate sets of modes develop with the maxima of the energy beam modulation supporting a set of shorter wavelength modes than those of the modulation minima by a factor $\Delta \lambda / \lambda \approx 4 \gamma_{m} / \gamma_{0}$. These are observed in the spectrum inset of Fig. 3 as a splitting of the spectrum at each of the main mode wavelengths, although other effects will be present which will cause further structure in the spectrum due to the energy chirp of the beam. The electron beam bunching parameter is also observed to have a similar pulse train structure as that of the radiation.

\section{ROLE OF ENERGY MODULATION}

In Sec. II C, the amplitude of the energy modulation was chosen by performing simulations with different modulation amplitudes and selecting the optimum for pulse train generation. Radiation pulse trains were shown to develop at the positions of minimum beam energy gradient at the extrema of the energy modulation. Here, the role of energy modulation in the mode-locked amplifier is considered further. To avoid noise in the temporal properties associated with SASE, a MLOK FEL amplifier simulation was seeded using a model developed to describe an attosecond pulse train from a high harmonic source. ${ }^{14}$

Previous studies ${ }^{14}$ have shown that the pulse train structure of an $\mathrm{HH}$ source can be retained during amplification to saturation in the mode-locked FEL without modulation of the electron beam. However, the radiation pulses broaden as the FEL interaction progresses. Here, it is shown that an electron beam energy modulation can restrict this pulse broadening effect, and a novel method for seeding the modelocked configuration is proposed which requires no phase matching between the seed and modulated beam.

\section{A. Modelling method}

Simulations were carried out assuming a uniform electron beam current using a 1-D code similar to that described in Ref. 22. The notation follows that of Ref. 12, with the scaled units of Refs. 3 and 4 being used. In these units, $\bar{z}_{1}$ is the local distance measured within the electron beam in units of the cooperation length, $l_{c}$. The relative propagation, or "slippage," of a radiation wavefront with respect to the electron bunch per undulator-chicane module in these units is $\bar{s}=\bar{l}+\bar{\delta}$, where $\bar{l}$ is the slippage in the undulator $\left(\bar{l}=N_{u} \lambda_{r} / l_{c}\right)$ and $\bar{\delta}$ is that within the chicane. The scaled resonant FEL frequency is $\bar{\omega}=\left(\omega-\omega_{r}\right) / 2 \rho \omega_{r}$ and the slippage enhancement factor is $S_{e}=\bar{s} / \bar{l}$.

The temporal profile of the HH seed is given by a comb of attosecond pulses separated by half the wavelength of the drive laser, $\lambda_{d}$. The HH seed was modelled in a similar way to that described in Refs. 14 and 23 with the resonant FEL wavelength chosen to be the 65 th harmonic of the drive laser, i.e., $\lambda_{r}=\lambda_{d} / 65$. For a typical drive laser wavelength of $\lambda_{d}=805 \mathrm{~nm}$ (e.g., Ti:Sapphire), the 65th harmonic is 12.4 $\mathrm{nm}$. The temporal and modal structure used to model the seed is shown in Fig. 5.

With a FEL parameter of $\rho=2 \times 10^{-3}$ (typical for an FEL operating in the extreme ultraviolet (XUV) and with 8 periods per undulator module, the scaled undulator slippage is $\bar{l}=0.201$. The spectral and temporal structure of the radiation generated by the undulator-chicane system was matched to the $\mathrm{HH}$ seed by setting the scaled chicane slippage to be $\bar{\delta}=0.616$ so that $\bar{s}=\bar{\lambda}_{d} / 2$, where $\bar{\lambda}_{d}=\lambda_{d} / l_{c}$ is the scaled drive laser wavelength, giving a slippage enhancement factor of $S_{e} \approx 4.1$.

\section{B. Beam modulation and seed phase-matching}

An electron beam energy modulation was introduced of period $\bar{\lambda}_{d} / 2$ equal to both the slippage in an undulatorchicane module and the $\mathrm{HH}$ seed pulse train spacing. Figure 6 shows a schematic of two different initial phases of the $\mathrm{HH}$ seed with respect to the beam energy modulation phase. The subsequent slippage of a radiation wavefront relative to the 

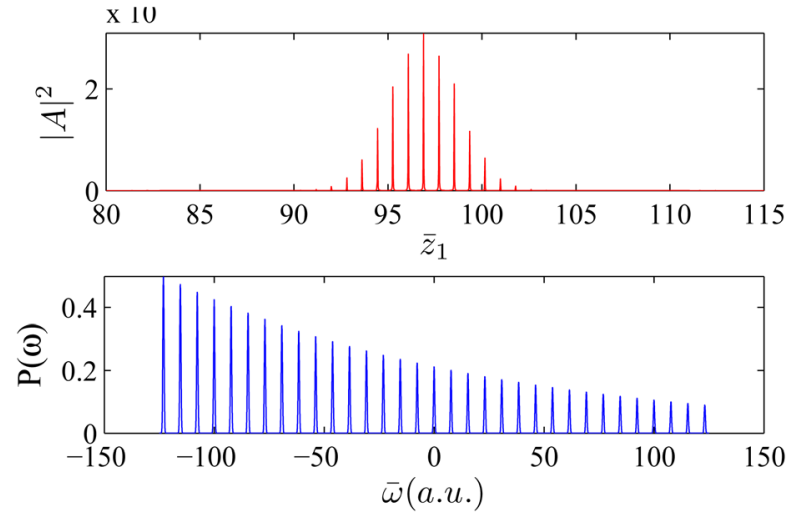

FIG. 5. (Color online) Scaled longitudinal intensity profile (top) and spectral power distribution (bottom) for the $\mathrm{HH}$ seed.

electron beam due to that occurring in the undulators and chicanes are also shown. It is clear that the FEL interaction in the undulators will differ for the two initial phases due to the different electron energy chirps experienced by the radiation pulses.

The initial phase $\bar{z}_{10}$ of a peak of the HH seed pulse with respect to the beam energy modulation was varied over one modulation period and the scaled power of the radiation recorded for fixed undulator length $\bar{l}$. The results are plotted in Fig. 7 for a range of energy modulation amplitudes. For zero energy modulation, there is clearly no dependence on the initial phase $\bar{z}_{10}$ of the seed. As the amplitude increases, the initial phase alignment of the seed becomes more significant. The optimum amplification of the seed was seen to occur with seed pulses phased $\bar{l} / 2$ behind the maxima and minima of the energy modulated electron beam at the beginning of each undulator as shown in Fig. 6(b). This

(a)

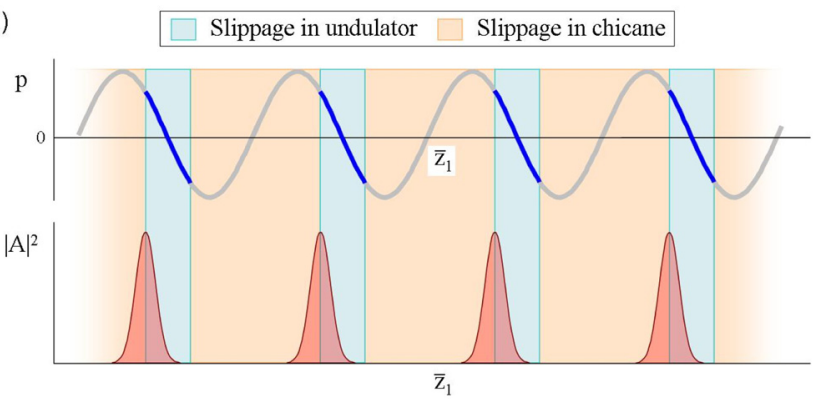

(b)

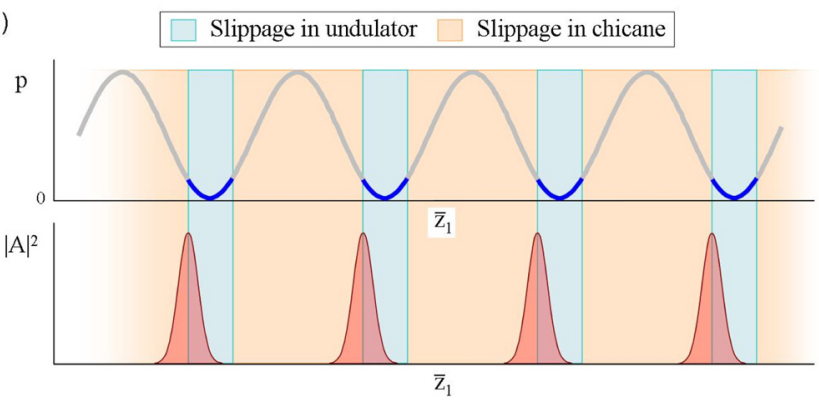

FIG. 6. (Color online) Alignment of the $\mathrm{HH}$ seed pulses relative to the electron beam energy modulation at the start of an undulator module for two cases (a) $\bar{l} / 2$ behind the central energy of the modulation and (b) $\bar{l} / 2$ behind the minima of the energy modulation. Here, $p=\left(\gamma-\gamma_{0}\right) / \rho \gamma_{0}$ is the scaled electron beam energy.

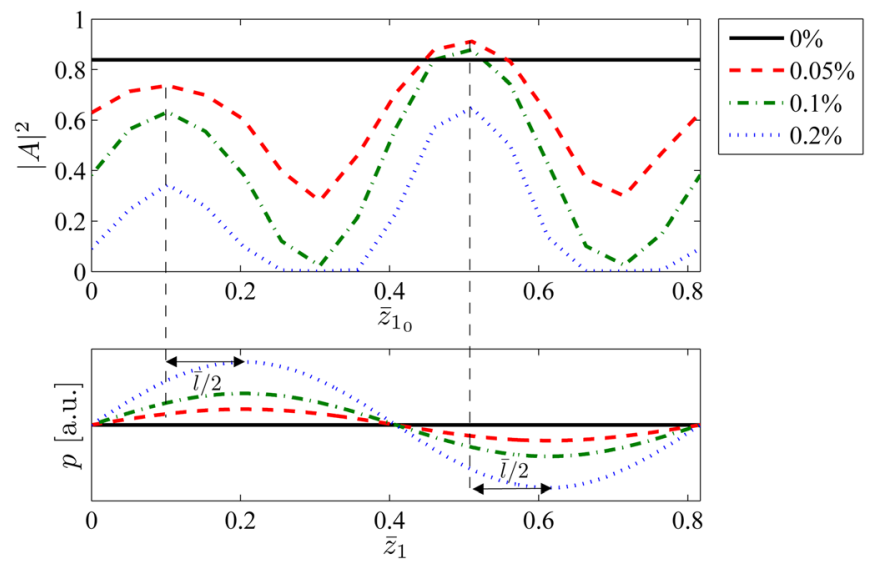

FIG. 7. (Color online) Peak radiation intensity after 40 modules against initial longitudinal position of the seed pulses (top) relative to the electron beam energy modulation (bottom), for varying energy modulation amplitude. The initial alignment of the seed becomes more critical as the energy modulation amplitude increases. The optimum initial alignment of the peak powers of the seed was found to be half of the undulator slippage behind the positions of zero energy gradient.

corresponds to the optimum radiation peak powers being coincident with the zero energy gradient of the electron beam at the middle of each undulator module and concurs with the results of the start-to-end simulations of Sec. III C where the amplification started from noise.

For larger beam energy modulation amplitudes, it may be expected that those regions of the beam that have sufficiently large energy gradients, such as Fig. 6(a), will not support FEL lasing. Assuming an energy modulation of the form

$$
\gamma=\gamma_{0}+\gamma_{m} \cos \left(\Delta \bar{\omega} \bar{z}_{1}\right)
$$

then a radiation wavefront propagating about the maximum of the beam energy gradient will experience a beam energy range of $\Delta \gamma \approx \gamma_{m} \Delta \bar{\omega} \bar{l}$ in one undulator module. If this range is sufficiently large that $\Delta \gamma / \gamma_{0}>\rho$, then the FEL interaction will be suppressed. ${ }^{3}$ Hence, for a beam modulation period defined by the radiation mode spacing, $\Delta \bar{\omega}=2 \pi / \bar{s}$, then, for beam energy modulation amplitudes,

$$
\gamma_{m} / \gamma_{0}>\rho S_{e} / 2 \pi
$$

the radiation peak powers will start to become confined to grow about to the extrema of the beam energy modulation where the energy gradients are smallest. For the above parameters used here, this corresponds to $\gamma_{m} / \gamma_{0}>0.13 \%$. The effect can be seen from Fig. 7 where radiation output is suppressed with increasing beam energy modulation amplitude for pulses that are phased to evolve in the regions of high energy gradient about $\bar{z}_{1_{0}} \approx 0.3$ and 0.7 .

The optimal longitudinal phase alignment of the seed of $\bar{z}_{10}=0.51$ from Fig. 7 was used in the simulations and the energy modulation amplitude varied. The scaled pulse width $\bar{l}_{r}$ and the peak scaled radiation power $|A|_{\text {peak }}^{2}$ are plotted as a function of the interaction distance through the undulator $\bar{z}$, in Figure 8. With zero energy modulation, the radiation pulses are seen to broaden in agreement with previous studies. ${ }^{14}$ An energy modulation amplitude of $0.1 \%-0.2 \%$ is 

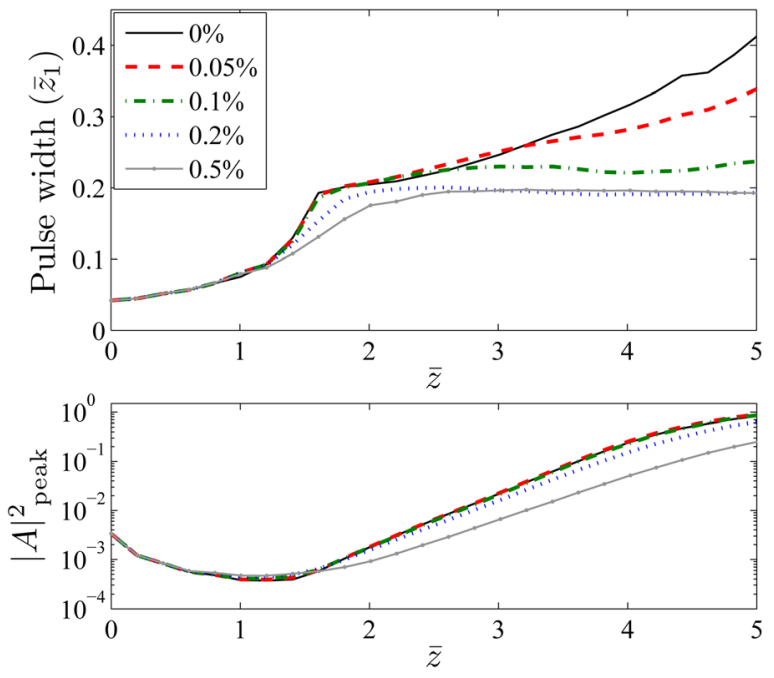

FIG. 8. (Color online) Scaled radiation pulse width $\bar{l}_{r}$ (top) and peak intensity $|A|_{\text {peak }}^{2}$ (bottom) plotted as a function of scaled distance, $\bar{z}$, through the undulator for different energy modulation amplitudes. The optimal initial seed alignment is used $\left(\bar{z}_{1_{0}} \approx 0.51\right.$ in Fig. 7). An energy modulation amplitude of $\gamma_{m} / \gamma_{0} \approx 0.2 \%$ is sufficient to restrict pulse broadening. Increasing the modulation amplitude further does not reduce the pulse width but does reduce the growth rate.

seen to be sufficient to restrict the pulse broadening effects in accord with condition (2). Increasing the modulation amplitude above this value does not reduce the output pulse width further, which has a lower limit determined by the relative slippage in one undulator module, to give $\bar{l}_{r} \approx \bar{l}=0.2$. Increased beam energy modulation amplitude does however decrease the radiation growth in accord with the condition (2).

A condition for the upper limit of the energy modulation that accommodates the dual criteria of allowing sufficient growth rate while minimising the pulse duration may be obtained by considering the beam energy range a radiation pulse interacts with when propagating $\pm \bar{l} / 2$ about the extrema of the modulated beam. By expanding $\cos (x) \approx 1-$ $x^{2} / 2$ in the modulation term of Eq. (1), and letting $\Delta \bar{z}_{1}=\bar{l} / 2$, then setting the energy range to the upper limit of $\rho$, the condition for the upper limit of the energy modulation is obtained

$$
\gamma_{m} / \gamma_{0}<2 \rho S_{e}^{2} / \pi^{2}
$$

For the parameters used above, this corresponds to a beam energy modulation of $\gamma_{m} / \gamma 0<0.68 \%$.

The conditions $(2,3)$ may be combined to give the range of electron beam energy modulation that allow the pinning of the radiation pulses to the beam modulation extrema while allowing for a satisfactory radiation growth rate

$$
\frac{S_{e}}{2 \pi}<\frac{\gamma_{m}}{\rho \gamma_{0}}<\frac{2 S_{e}^{2}}{\pi^{2}}
$$

The difference in gain between the maxima and minima of the energy modulations is noted with the minima (positive energy curvature) giving slightly greater gain, however further analysis is required to fully understand this effect.
While in the HH seeded MLOK FEL, the seed pulses are optimally amplified when aligned to the positions of minimum beam energy gradient at the extrema of the modulation. In the SASE mode, when the system starts from noise, the extrema now define the positions about which the radiation pulses preferentially grow as observed in the start-toend simulations of Sec. III A. This pinning of the radiation pulses to these well defined phases, in both the seeded and SASE cases, is of significant benefit where the phasing of the output pulses to an external signal is important e.g., in pump-probe type experiments.

\section{Filtered seed}

The Sec. III B showed that use of an HH seed allows control of the envelope and relative phases of the radiation pulse train over lengths significantly greater than the cooperation length, and that pulse broadening effects ${ }^{14}$ could be reduced by seeding an energy modulated beam. However, phase-matching of the $\mathrm{HH}$ seed pulse train to the correct phase of the electron beam energy modulation was necessary to achieve optimum output. It is now shown that it is possible to seed the MLOK FEL with an HH source that is filtered to remove all harmonics except the resonant harmonic of the central mode. As this filtering removes the attosecond pulse train structure of the seed, no phase-matching of seed and energy modulated beam is required at the start of the interaction. However, the temporal coherence of the seed is retained during amplification and the mode-locked pulse train structure grows as the interaction proceeds.

The HH seed field of Fig. 5 was filtered to preserve only the central resonant mode, $\bar{\omega}=0$, removing the attosecond structure so that the seed retains the simple Gaussian envelope of the $\mathrm{HH}$ drive laser. A beam energy modulation amplitude of $\gamma_{m} / \gamma_{0}=0.2 \%$ was used in these simulations. The minimum of the modulated beam energy, with the larger growth rate, was chosen to be resonant with the seed. Other parameters are similar to those used in Sec. III B and results of the simulations are shown in Fig. 9. While the interaction was seeded at the central mode frequency only, the mode frequencies quickly develop, lock, and were amplified to generate a pulse train which retains the good temporal coherence properties of the seed. At saturation similar pulse widths and powers to those of Fig. 8 of Sec. III B were obtained.

\section{SIMULATION MODEL LIMITATIONS AND POTENTIAL FUTURE DEVELOPMENT}

Most computational codes that model the FEL interaction, like those used in the results presented above, assume that the simulation electrons are confined to their initial localised ponderomotive potentials and that the FEL interaction is averaged over an undulator period. For some parameters, these approximations may not be valid when simulating FEL interactions that utilise energy modulated electron beams, such as the MLOK.

For a sufficiently large energy modulation amplitude of the electron beam, longitudinal electron transport on scales greater than the resonant radiation wavelength cannot be modelled easily using simulation codes such as those used in 

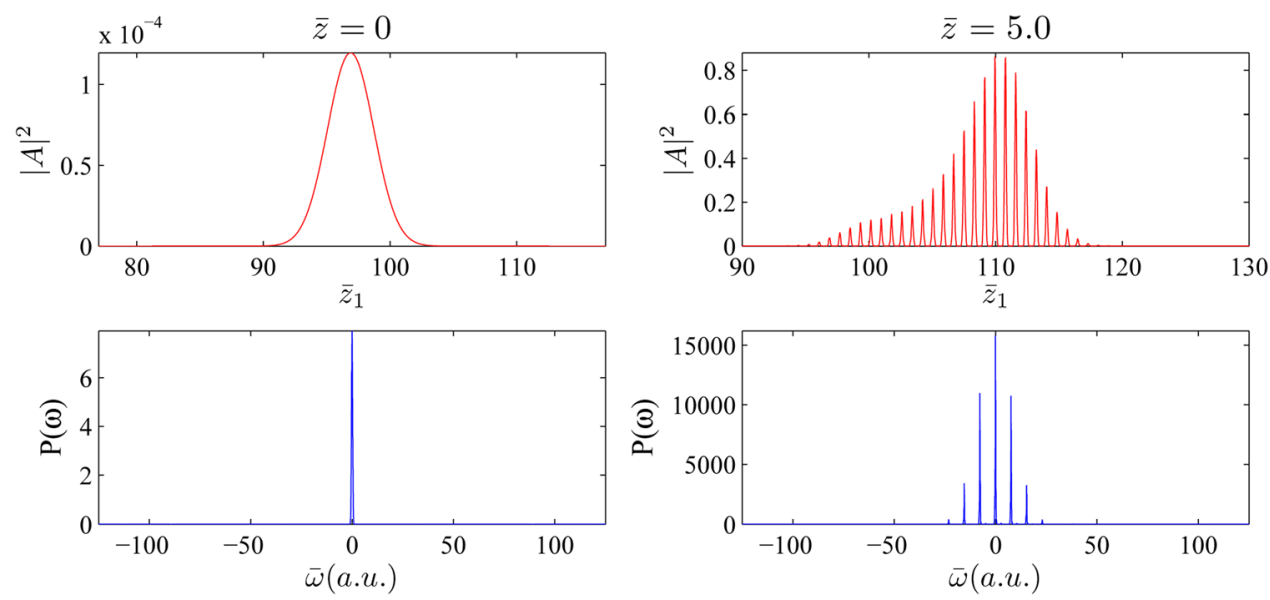

FIG. 9. (Color online) Simulation results for the MLOK FEL with a filtered, single mode, HH seed. The left plots show the initial scaled power (top) and scaled spectral power (bottom) of the seed at $\bar{z}=0$. The right plots show the equivalent close to saturation at $\bar{z}=5$. this paper. On propagation through the undulator-chicane modules, dispersion will cause beam energy modulation, with a period of the radiation mode spacing, to develop into a density (current) modulation of the same period. While this current modulation will not affect the creation of the radiation modes, which is essentially an optical process, the current modulation can by itself couple and lock the radiation modes to generate a train of short pulses. ${ }^{24}$ This effect may, therefore, be beneficial to the mode-locking process. However, to investigate these effects will require new simulation methods that allow for beam current evolution as the interaction progresses. In order for such dispersive effects to be small, as required by the simulation codes used in this paper, the growth rate of the current modulation should be significantly smaller than the FEL interaction in a gain length $l_{g}$, and a condition that describes this is now derived.

From Ref. 12, the effect of chicane dispersion on the scaled position of the $j$ th electron with scaled energy $p_{j}=\left(\gamma_{j}-\gamma_{0}\right) / \rho \gamma_{0}$ is given by

$$
\bar{z}_{1 j} \rightarrow \bar{z}_{1 j}+2 \rho D p_{j},
$$

where $D=R_{56} / 2 l_{c} \approx 10 \bar{\delta} / 6$ is the scaled dispersive strength of a single chicane. The dispersive effects can act to enhance the FEL electron bunching process at the radiation wavelength so reducing the gain and saturation lengths in what has been called a distributed optical klystron. ${ }^{25-27}$ However, these dispersive effects will also have an effect on a beam with energy modulation of period greater than the radiation wavelength, such as that employed to couple the modes in the MLOK FEL. In this case, the dispersive bunching would maximise for an approximate propagation in $\bar{z}_{1}$ of $\bar{s} / 4$ by the maximum and $-\bar{s} / 4$ by the minimum of energy modulation. The effects of such bunching may be considered negligible if it is much less than the modulation period in a FEL saturation length, which may be written as the condition

$$
2 \rho N_{s a t} D p_{m} \ll \frac{\bar{s}}{4}
$$

where $N_{\text {sat }}=\bar{z}_{\text {sat }} / \bar{l}$ is the number of undulator chicane modules in saturation length $\bar{z}_{s a t}$ and $p_{m}=\gamma_{m} / \rho \gamma_{0}$ is the scaled beam energy modulation amplitude. The definition of the slippage enhancement factor $S_{e}=\bar{s} / \bar{l}$ and the relation above for the scaled dispersive strength $D$, may be used to obtain

$$
\frac{\gamma_{m}}{\gamma_{0}} \ll \frac{3}{40 N_{s a t}} \frac{S_{e}}{S_{e}-1} .
$$

In the start-to-end simulations, using the tracked electron beam of Sec. II, this inequality is satisfied by a factor of approximately four, while for the simulations of Sec. III, it is satisfied by a factor of approximately two for the $\gamma_{m} / \gamma_{0}=0.2 \%$ modulation amplitude case. Some effects due to dispersion on the energy modulated beam may, therefore, be expected. Such effects, as now discussed, may in fact be beneficial to radiation generation.

Modulation of the electron beam energy, and the current modulation that results, can also be a significant source of coherent spontaneous emission (CSE), another process not modelled by averaged codes. ${ }^{28}$ Initial studies ${ }^{29}$ indicate that this is the case and that the increased frequency content able to be to be modelled (due to a larger Nyquist frequency) introduces further, shorter duration, pulse structure. This may occur without any collective high-gain FEL interaction, i.e., relying on CSE only, and the MLOK may be able to generate pulse trains from beams that may not be able to operate as an FEL e.g., due to deleterious beam quality effects. This may open up new opportunities for light sources and will be the subject of future research.

\section{CONCLUSIONS}

The MLOK FEL configuration has been shown to be compatible with the predicted output from the present generation of accelerators by using so-called start to end numerical simulations. The role of electron beam energy modulation has been described and explains the relative phasing of the radiation pulse evolution with respect to the beam energy modulation. A relatively simple method of seeding the MLOK FEL with a filtered HH source was shown to control the envelope and phase of the mode-locked pulse train. Such control would assist in the phasing of the short pulse train 
with respect to a pump field in pump-probe type experiments. The limitations of present, averaged, FEL simulation codes were considered and a criteria for the validity of these codes derived when energy modulated beams are employed. Finally, the use of coherent spontaneous emission in a MLOK configuration was discussed. Such systems may offer a new method of generating short pulse trains of coherent, high power radiation without the need to rely upon the FEL interaction.

\section{ACKNOWLEDGMENTS}

This work received support from STFC Memorandum Of Agreement Number 4163192.

${ }^{1}$ P. Emma, R. Akre, J. Arthur, R. Bionta, C. Bostedt, J. Bozek, A. Brachmann, P. Bucksbaum, R. Coffee, F.-J. Decker, Y. Ding, D. Dowell, S. Edstrom, A. Fisher, J. Frisch, S. Gilevich, J. Hastings, G. Hays, Ph. Hering, Z. Huang, R. Iverson, H. Loos, M. Messerschmidt, A. Miahnahri, S. Moeller, H.-D. Nuhn, G. Pile, D. Ratner, J. Rzepiela, D. Schultz, T. Smith, P. Stefan, H. Tompkins, J. Turner, J. Welch, W. White, J. Wu, G. Yocky, and J. Galayda, Nature Photon. 4, 641 (2010).

${ }^{2}$ B. W. J. McNeil and N. R. Thompson, Nature Photon. 4, 814 (2010).

${ }^{3}$ R. Bonifacio, C. Pellegrini, and L. Narducci, Opt. Commun. 50, 373 (1984).

${ }^{4}$ R. Bonifacio, B. W. J. McNeil, and P. Pierini, Phys. Rev. A 40, 4467 (1989).

${ }^{5}$ R. Bonifacio, L. de Salvo, P. Pierini, N. Piovella, and C. Pellegrini, Phys. Rev. Lett. 73, 70 (1994).

${ }^{6}$ E. L. Saldin, E. A. Schneidmiller, and M. V. Yurkov, Phys. Rev. ST Accel. Beams 9, 050702 (2006).

${ }^{7}$ A. A. Zholents and M. S. Zolotorev, New J. Phys. 10, 025005 (2008).

${ }^{8} \mathrm{P}$. Emma, Z. Huang, and M. Borland, in Proceedings of the 26th International FEL Conference (Trieste, 2004), p. 333. Available from Joint Accelerator Conferences online at http://www.jacow.org.

${ }^{9}$ P. B. Corkum and F. Krausz, Nat. Phys. 3, 381 (2007).

${ }^{10}$ F. Krausz and M. Ivanov, Rev. Mod. Phys. 81, 163 (2009).

${ }^{11}$ D. Xiang, Z. Huang, and G. Stupakov, Phys. Rev. ST. Accel. Beams 12, 060701 (2009).

${ }^{12}$ N. R. Thompson and B. W. J. McNeil, Phys. Rev. Lett. 100, 203901 (2008).

${ }^{13}$ B. W. J. McNeil and N. R. Thompson, "Mode locked optical klystron configuration in an FEL cavity resonator, THOB4," in Proceedings of the 32nd International FEL Conference (Malmö, Sweden, 2010), pp. 558561.

${ }^{14}$ B. W. J. McNeil, N. R. Thompson, D. J. Dunning, and B. Sheehy, J. Phys. B 44, 065404 (2011)

${ }^{15}$ P. H. Williams, B. D. Muratori, S. L. Smith, J. Rowland, D. AngalKalinin, J. K. Jones, H. L. Owen, R. Bartolini, and I. P. Martin, "A recirculating linac as a candidate for the UK New Light Source, WE5RF," in 23rd Particle Accelerator Conference (Vancouver, Canada, 2009). Available from Joint Accelerator Conferences online at http://www.jacow.org.

${ }^{16} \mathrm{~J}$.-H. Han, H. Huang, and S. Pande, "Technical design of the baseline gun for the NLS project, TUPC44," in Proceedings of the 31st International FEL Conference (Liverpool, UK, 2009), pp. 340-343.

${ }^{17}$ K. Floettmann, ASTRA Users Manual, available at http://www.desy.de/ mpyflo/Astra_dokumentation/ (Deutsches Elektronen-Synchrotron, Hamburg, Germany).

${ }^{18}$ M. Borland, User's Manual for elegant, available at http://www.aps. anl.gov/Accelerator_Systems_Division/Accelerator_Operations_Physics/ manuals/elegant_latest/elegant.html (Argonne National Laboratory, Argonne, USA).

${ }^{19} \mathrm{R}$. Soliday and M. Borland, elegant2genesis, available at http://www. aps.anl.gov/Accelerator_Systems_Division/Operations_Analysis/manuals/ SDDStoolkit/node32.html (Argonne National Laboratory, Argonne, USA).

${ }^{20}$ S. Reiche, Nucl. Instrum. Methods Phys. Res. A 429, 243 (1999).

${ }^{21}$ NLS Project: Conceptual Design Report, edited by J. Marangos and R. Walker (2010), available at http://www.newlightsource.org (Science and Technology Facilities Council, UK).

${ }^{22}$ B. W. J. McNeil, G. R. M. Robb, D. Dunning, and N. R. Thompson, "FELO: A one-dimensional time-dependent FEL oscillator code, MOPPH011," in Proceedings of the 28th International FEL Conference (Berlin, Germany, 2006), pp. 59-62. Available from Joint Accelerator Conferences online at http://www.jacow.org.

${ }^{23}$ B. W. J. McNeil, J. A. Clarke, D. J. Dunning, G. J. Hirst, H. L. Owen, N. R. Thompson, B. Sheehy, and P. H. Williams, New J. Phys. 9, 82 (2007).

${ }^{24}$ E. Kur, D. J. Dunning, B. W. J. McNeil, J. Wurtele, and A. A. Zholents, "A wide bandwidth free-electron laser with mode locking using current modulation," New J. Phys. 13, 063012 (2011).

${ }^{25}$ V. N. Litvinenko, Nucl. Instrum. Methods Phys. Res. A 304, 463 (1991).

${ }^{26}$ G. R. Neil and H. P. Freund, Nucl. Instrum. Methods Phys. Res. A 475, 381 (2001).

${ }^{27}$ Y. Ding, P. Emma, Z. Huang, and V. Kumar, Phys. Rev. ST Accel. Beams 9, 070702 (2006).

${ }^{28}$ B. W. J. McNeil, M. W. Poole, and G. R. M. Robb, Phys. Rev. ST Accel. Beams 6, 070701 (2003)

${ }^{29}$ D. Dunning, B. McNeil, N. Thompson, and B. Sheehy, Workshop on highharmonic seeding for present and future short wavelength Free-Electron Lasers (FELs), Frascati, Italy, 2008, available at http://www.elettra. trieste.it/FERMI/index.php?n=Main.SeedingWS. 